\title{
The Use of Platelet-Rich Fibrin in Bone Grafting
}

\author{
Belir Atalay and Ozge Doganay \\ Additional information is available at the end of the chapter \\ http://dx.doi.org/10.5772/intechopen.79825
}

\begin{abstract}
In this century, there are two mostly practiced techniques for bone regeneration. These are autogenous bone grafting (ABG) and guided bone regeneration (GBR). It was reported in the late 1970s that platelets have a good regenerative effects. Platelets include growth factors that increase vascularization and collagen production by cell mitosis. Recently, most of the studies have indicated that platelet-rich fibrin (PRF) is a great healing potential for bony and soft tissue that derived from patients own blood. Most beneficial effects of PRF are easily derived directly from patient's venous blood without any ingredients, and it has a great potential for hard and soft tissue regeneration. PRF has no inflammatory effects and can be used with all kind of graft materials. When used as a membrane, it helps protecting the surgical area to stimulate the healing of soft and bone tissues.
\end{abstract}

Keywords: platelet-rich fibrin, bone healing, oral surgery, regeneration

\section{Introduction}

Platelet concentrates (PCs) for surgical use are innovative tools of regenerative medicine and dentistry and were widely studied in oral and maxillofacial surgery. PCs are blood extracts, applied to surgical sites, injuries, or wounds, a safe and effective way to promote soft tissue healing and bone growth. The most frequently used substrate, platelet-rich fibrin (PRF) is a member of platelet concentrates developed by Choukroun and colleagues [1]. PRF is an autologous matrix, in which cytokines and cells are released after a short period [2].

The aim of this chapter is to describe the potential functions of PRF for bone grafting in oral and maxillofacial surgery.

There are different types of PRF in local use. 


\section{Pure platelet-rich fibrin (P-PRF)}

Pure platelet-rich fibrin (P-PRF) in other terms leukocyte-poor platelet-rich fibrin is a preparation without leukocytes having a high-density fibrin network. This platelet concentrate exists in a strongly activated gel form. It cannot be injected and used like traditional fibrin glues. On the other hand, with its strong fibrin matrix facility, P-PRF can be handled like a real solid material for several applications [3].

During preparation, only very low amounts of leucocytes are collected because of the specific separator gel, which is used to obtain P-PRF. However, the platelet collection level is high, and the preservation of the platelets during the procedure seems to be acceptable [4]. The main disadvantage of this platelet concentrate protocol is its cost and complex procedure if it is compared to the other forms of PRF, which are available nowadays [3].

\section{Leukocyte and platelet-rich fibrin (L-PRF)}

The second platelet concentrate type is leukocyte and platelet-rich fibrin (L-PRF), which is known as leukocyte and platelet-rich fibrin concentrate, called Choukroun's PRF. This product was invented by Choukroun in 2000 [1, 2]. The main concept of this technique differs from the other protocols. Patient's blood is taken and immediately softly centrifuged. This provides the formation of a fibrin clot in the middle of the tube, between the red blood cell base at the bottom and the acellular plasma at the top. This clot includes nearly all the platelets and more than $50 \%$ of the leukocytes from the initial blood harvest $[5,6]$. The high quantity of leukocytes provides immune and antibacterial properties, wound healing and growth factor regulation. But, it depends on which leukocytes, in which quantity and in which state the centrifugation process can softly activate the white blood cells [7]. This product therefore only exists in an activated form and cannot be injected like a suspension. Therefore, L-PRF is a practical solid material with strong fibrin scaffold and used in oral and maxillofacial surgery, periodontology, implant dentistry and ear nose throat surgery [6].

\section{Advanced platelet-rich fibrin (A-PRF)}

The amount of WBCs effect vascularization and bone remodeling. As a result, researchers made new modifications in the centrifugation speeds and times to prevent cell loss within the PRF matrix [8]. These recent modifications of the PRF protocol have led to the improvement of advanced platelet-rich fibrin (A-PRF), which uses lower G-forces to gain higher growth factors compared to PRF [9]. It maintains higher amount of WBCs in the fibrin matrix and has special glass tubes, which are designed to make clotting faster. After the centrifugation, the tubes are removed and placed in their holders and left for $5 \mathrm{~min}$ to start clot formation. The fibrin clot which is rich with WBCs provides higher growth factors and recent research emphasizes that A-PRF enhances collagen matrix synthesis and supplement of progenitor cells [8]. 


\section{Injectable platelet-rich fibrin (i-PRF)}

One of the recent developments in the PRF technology is injectable PRF (i-PRF). Standard PRF is prepared as a gel which is inconvenient to inject [10]. i-PRF protocol necessitates short centrifugation period in order to produce a liquid platelet concentrate, which includes primarily liquid fibrinogen and thrombin prior to fibrin formation [11]. The plastic tubes with hydrophobic surface are used, and therefore coagulation process does not effectively start. Hence, all the blood components reach the top of the tube under the centrifugation force with short centrifugation time (i.e., 2-4 min). The light yellow-colored layer, which is the combination of plasma and platelets, is aspirated from the top of the tube to obtain i-PRF. Nowadays, it is used with bone grafts to keep graft particles tightly encapsulated in the fibrin matrix. With the coagulation process, i-PRF forms a gel consistency holding bone graft together. Also, the release of growth factor is beneficial for the graft. This has the potential to convert any osteoconductive graft to osteopromotive, which would provide faster and better bone formation. Another type of graft prepared with i-PRF is the PRF block. For its preparation, i-PRF is mixed with a combination of bone graft and shredded PRF clot [10].

\section{Titanium prepared platelet-rich fibrin (T-PRF)}

Some physicians worry about damage for the patient with glass-evacuated blood collection tubes with silica activators. O'Connell emphasized that silica contact cannot be avoided with glass tubes. These silica particles are dense enough to settle in the sediment and might reach the patient when the product is used during procedures [12]. Recently, Tunali et al. used medical grade titanium tubes to produce titanium prepared platelet-rich fibrin (T-PRF) [10]. This biocompatible material was tried to eliminate the potential negative effects of silica from dry glass or glass-coated plastic tubes [12]. The research showed that T-PRF supply is a more organized network than L-PRF. Furthermore, its fibrin network covers larger area and has thicker fibrin clot. In a human study, wound healing in the palatal mucosa is found better with the T-PRF application [10].

\section{Prepared platelet-rich lysate (PRF-L)}

A newer application of PRF is the prepared platelet-rich lysate (PRF-L). In this technique, after PRF preparation, it is incubated at $37^{\circ} \mathrm{C}$ in a humidified atmosphere of $5 \% \mathrm{CO}_{2}$ and $95 \%$ air. The exudate, which is collected, is referred to as PRF lysate. It is said to be a good source of several growth factors. In a study, it is found that PRF-L can significantly improve the proliferation index, collagen deposition and migration rates in chronically UVA-irradiated human dermal fibroblasts [13]. This is a new technique that requires further studies. 


\section{Preparation}

Venous blood of patient is drawn into $10 \mathrm{~mL}$, anticoagulant-free tubes and then immediately spun in a tabletop centrifuge at $3000 \mathrm{rpm}$ for $10 \mathrm{~min}$. Due to the lack of anticoagulant, platelet activation occurs once the blood contacts the glass tube walls and subsequently initiates the coagulation cascade. By centrifugation, fibrinogen is concentrated in the top and middle of the tube and then circulating thrombin transforms it into fibrin. This action leads to the development of a fibrin clot in the middle of the tube, between the RBCs at the bottom and serum at the top. The upper straw-colored layer is removed from the centrifuge tube and blotted to remove serum and RBC to result in a dense matrix. Timing is also critical to the success of the PRF technique. PRF must be prepared immediately before application to the surgical site (Figure 1) [1].

\subsection{Clinical applications of PRF in oral surgery}

In the past, PRF could be useful for both hemostasis and wound healing. Nowadays, sinus lift technique is considered to be one of the most common and valid procedures for the use of PRF in oral surgery. PRF is also used for augmenting the alveolar ridge, improve soft tissue healing and periodontal regeneration and preserving the alveolar ridge height after extraction. It has been studied that PRF improves wound healing in multiple extractions by reducing healing time [14].

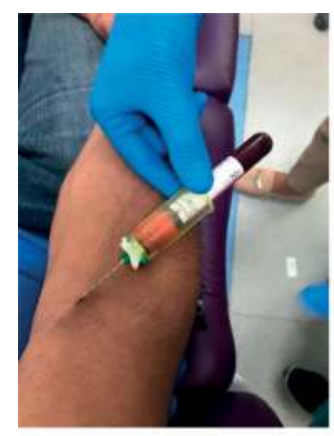

A

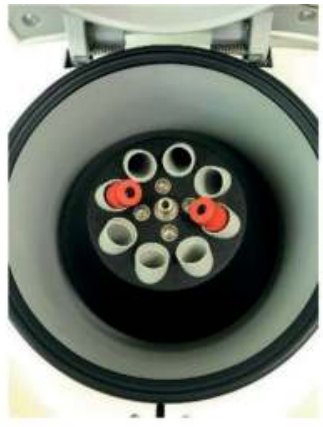

B

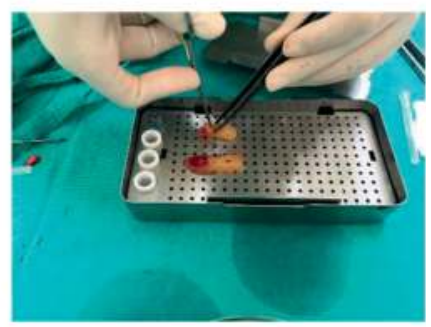

C

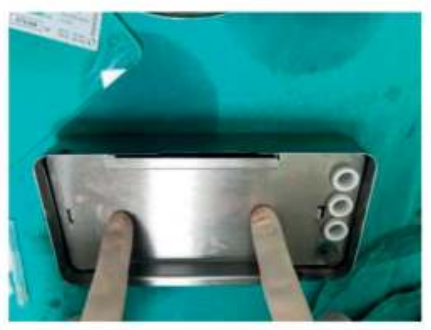

D

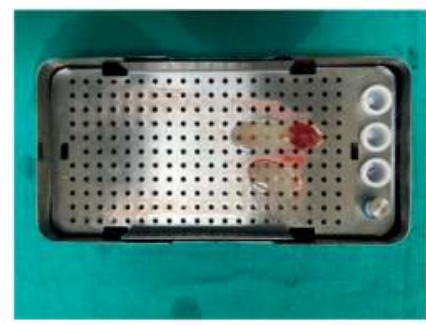

$\mathrm{E}$

Figure 1. Preparation of PRF membrane. A: Collection of venous blood into the tube; B: centrifuge device; C: platelet-rich fibrin clots; D: PRF clots were pressed to obtain more dense membranes; E: PRF membranes. 


\subsubsection{PRF in soft tissue regeneration}

The use of platelet concentrates may reduce and control the risks associated with delayed bone and soft tissue healing. The success for the healing of a bone graft is certainly related with intact soft tissue coverage.

Patients using antiresorptive therapies exhibit a reduced healing potential in bone and soft tissue that can lead to medication related osteonecrosis. In order to stimulate healing and obtain soft tissue coverage, PRF can be placed to the surgical or exposed area without tension. Even if there is a need for researches in this field, PRF presented valuable solutions till today [15].

The other indication of PRF usage is the method of socket preservation. When implant restoration is considered after extraction of a tooth, this must be kept in mind that the resorption process starts immediately. Thus, the volume of the socket, soft tissue closure and thickness of gingiva must be protected as possible as early, for a complete and quick bone regeneration. If the wound closure and remodeling are achieved properly, implant can be placed in the desired position [15].

\subsubsection{Guided bone regeneration (GBR) (as a barrier membrane)}

Several oral and maxillofacial surgical procedures have used membranes for guided bone regeneration (GBR). The potential applications of PRF in this field have been shown to be effective in different clinical situations. However, this method may not be superior as a barrier membrane for regeneration when compared to conventional membranes, and therefore appropriate cases should be selected in order to benefit from the properties of PRF in GBR (Figure 2) [16, 17].

\subsubsection{Sinus lift procedure}

Sinus lift is one of the most frequent grafting procedures in implantology and has proven to be a successful and predictable technique for providing bone height for implant placement. In the

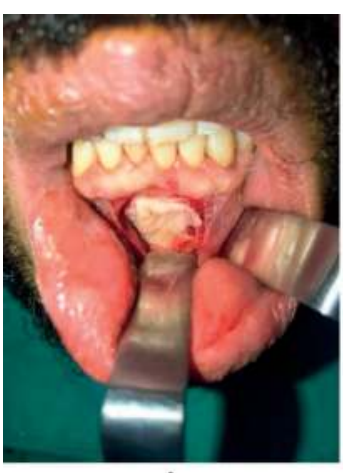

A

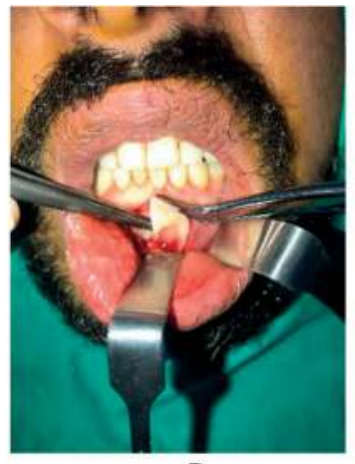

B

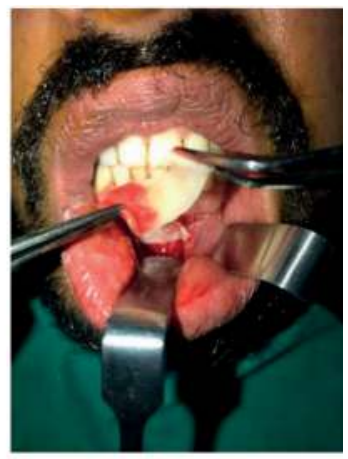

C

Figure 2. Enucleation of radicular cyst resulted from left central incisor tooth and apical resection were performed at the same time. A: After eliminating the whole lesions, PRF placed into the intrabony defect to accelerate healing and prevent soft tissue collapse. B and C: PRF membrane was used to cover the buccal wall of the cavity as a barrier. 
literature, no application using PRF was more studied than the sinus lift procedure. This model generating to obtain bone height, provides a closed, protected and stable environment for healing. Thus, bone healing in sinus cavity can be evaluated more accurate than the other procedures that are in contact with the oral environment [18].

Subsinus cavity floored by the Schneiderian membrane following sinus lift has a capacity of bone regeneration due to the high osteogenic properties, though several bone materials can yield good results in terms of bone healing [19].

Most researches have focused on bone biomaterials to improve bone quality and to accelerate healing for early operations. The main mechanism of subsinus cavity healing is to be filled with blood clot that is promoting neoangiogenesis and proliferation. Similarly, the aim of using PRF into the cavity is to provide space and optimize healing as a biologic agent [20]. The first study reported on PRF by Choukroun et al. represented that the use of PRF in addition to the bone grafts accelerated healing period for sinus floor augmentation [21]. It is important to reduce healing time before implantation.

The incidence of perforation of the membrane that is a common intraoperative finding was reported ranging from 10 to $60 \%$ [22]. The rupture of the membrane can lead to postoperative complications, so has to be repaired before grafting. Otherwise, a biomaterial can migrate into the sinus cavity leading to sinus infection and impairment of healing. Some biomaterials such as collagen membrane and platelet concentrates have been commonly used to prevent these complications. In addition to the use of PRF for grafting, it is also used as an adhesion material and frequently preferred for repair of possible perforations of the schneiderian membrane during sinus lift.

\subsubsection{Grafting material for bone regeneration}

The acceleration of healing in bone defect is of particular interest when dental implants would be inserted in this area. Due to reduction of time, early functional loading and better primary stability of the implant will be obtained after the regeneration [19]. Thus, some biologic agents may be needed to shorten this period. For this purpose, PRF-like substances can be used. The potential of these substances as a biologic agent in oral and maxillofacial surgery relies on the growth factors stored within platelet alpha granules containing platelet-derived growth factor (PDGF), vascular endothelial growth factor (VEGF), insulin-like growth factor (IGF), plateletderived angiogenic factor and transforming growth factor-beta (TGF- $\beta$ ). Moreover, PRF acts as a biological connector between different graft elements and serves as a matrix that eases forming new bone tissue into the defect. In fact, due to the lack of standardization and absence of characterization of the studied PRF materials, it is difficult to explain the exact function of platelet concentrates on bone regeneration [23].

\subsubsection{PRF for peri-implant bone defects}

Peri-implant bone defects are encountered as a result of peri-implantitis and secondly inadequate bone volume for implantation and thirdly immediate implantation. Although the mechanisms of peri-implantitis are considered to be bacterial accumulation or prosthetic overload of 
the implant, it is believed that the main mechanism of peri-implantitis is related to bone/ implant interface breakdown [24].

In case of small amount of bone, it is inevitable to make lateral perforation while placing implant or bone resorption around particularly the collar of the implant. In order to reinforce the bone amount around the implant to prevent resorption and gain more esthetic appearance, autogenous and/or synthetic materials can be used to cover the open surface of these implants.

Peri-implant bone defects can be developed after immediate implantation (Figure 3). After the insertion of the implant in a fresh avulsion socket, a large peri-implant defect must be filled with a material to prevent soft tissue invagination [25].

For those three clinical situations, bone regeneration between the cavity/defect and implant surface can be achieved by using PRF in addition to bone grafts. With its strong fibrin architecture and specific 3D cell distribution, especially L-PRF may offer positive results when used

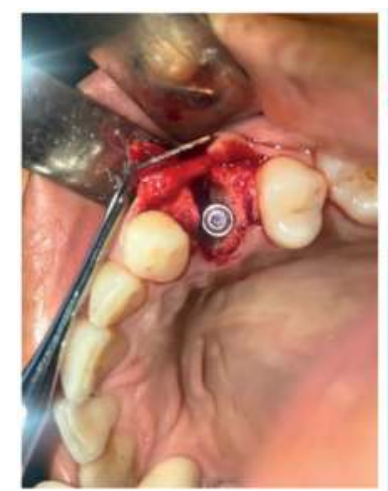

A

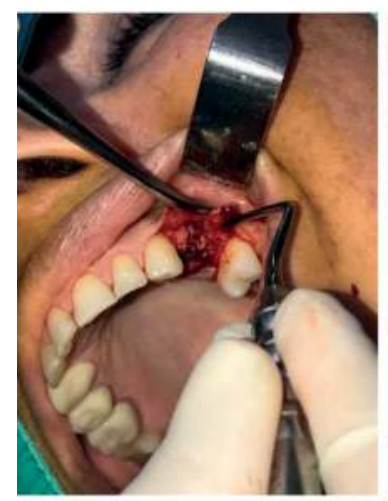

D

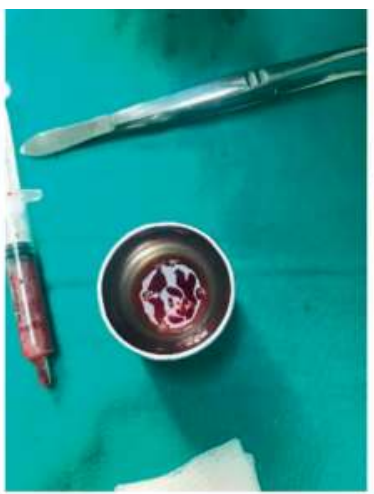

B

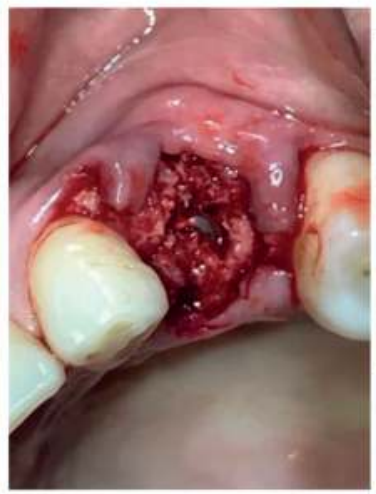

$E$

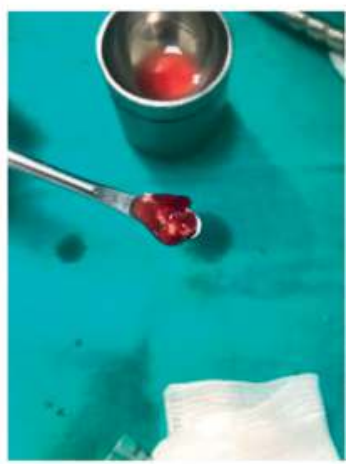

C

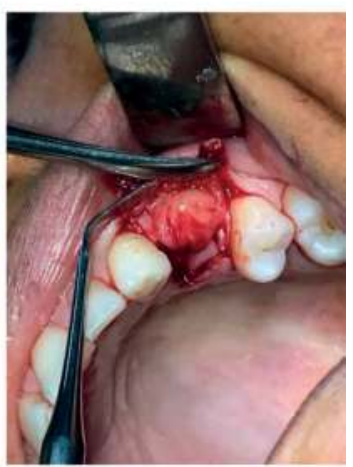

$\mathrm{F}$

Figure 3. Peri-implant bone defect after immediate implant placement. A: Buccal and palatinal bone defects around the implant; B, C: A mix of PRF and xenogeneic bone graft constituted a sticky mass; D, E: PRF with bone graft placed into the cavity; F: PRF membranes were used to cover the whole defect. 
in combination with bone biomaterials for the filling of peri-implant bone defects. On the whole, there are still only few studies published in the literature about the use of L-PRF in peri-implant bone defects [26].

\section{In brief: advantages}

- Eliminating transmission of diseases (the patient's own blood)

- $\quad$ Promoting soft tissue healing

- Protection extraction socket against resorption and infection

- Minimal invasive and inexpensive method

- $\quad$ Role as a carrier matrix for bone grafts

- Supporting neoangiogenesis

- Minimal immunological reaction

- As a sticky material, holding different substances together

- Growth factors

\section{Conclusion}

PRF seems to be an accepted minimally invasive approach with good clinical results. With the ease of preparation, low cost and biologic properties, PRF could be considered as a beneficial treatment option. On the other hand, due to the lack of standardization and long-term follow up clinical studies, the major effect of PRF on bone grafting has not been explained yet.

\section{Author details}

Belir Atalay $^{1}$ and Ozge Doganay ${ }^{2 *}$

*Address all correspondence to: ozgedoganay87@gmail.com

1 Department of Oral and Maxillofacial Surgery, Faculty of Dentistry, İstanbul University, İstanbul, Turkey

2 Department of Oral and Maxillofacial Surgery, Faculty of Dentistry, Bezmialem Vakıf University, İstanbul, Turkey 


\section{References}

[1] Dohan DM, Choukroun J, Diss A, et al. Platelet-rich fibrin (PRF): A second-generation platelet concentrate. Part I: Technological concepts and evolution. Oral Surgery, Oral Medicine, Oral Pathology, Oral Radiology, and Endodontics. 2006;101:e37-e44

[2] Dohan DM, Choukroun J, Diss A, et al. Platelet-rich fibrin (PRF): A second-generation platelet concentrate. Part II: Platelet-related biologic features. Oral Surgery, Oral Medicine, Oral Pathology, Oral Radiology, and Endodontics. 2006;101:e45-e50

[3] Ehrenfest DMD, Andia I, Zumstein MA, et al. Classification of platelet concentrates (platelet-rich plasma-PRP, platelet-rich fibrin-PRF) for topical and infiltrative use in orthopedic and sports medicine: Current consensus, clinical implications and perspectives. Muscles, Ligaments and Tendons Journal. 2014;4(1):3-9

[4] Ehrenfest DMD, Rasmusson L, Albrektsson T. Classification of platelet concentrates: From pure platelet-rich plasma (P-PRP) to leucocyte- and platelet-rich fibrin (L-PRF). Trends in Biotechnology. 2009;27(3):31

[5] Cano-Durán JA, Peña-Cardelles JF, Ortega-Concepción D, et al. The role of leucocyte-rich and platelet-rich fibrin (L-PRF) in the treatment of the medication-related osteonecrosis of the jaws (MRONJ). Journal of Clinical and Experimental Dentistry. 2017;9(8):e1051-e1059

[6] Ehrenfest DMD, Bielecki T, Jimbo R, et al. Do the fibrin architecture and leukocyte content influence the growth factor release of platelet concentrates? An evidence-based answer comparing a pure platelet-rich plasma (P-PRP) gel and a leukocyte- and platelet-rich fibrin (L-PRF). Current Pharmaceutical Biotechnology. 2012;13:1145-1152

[7] Fioravanti C, Frustaci I, Armellin E, et al. Autologous blood preparations rich in platelets, fibrin and growth factors. Oral Implantology. 2016 Jul 23;8(4):96-113

[8] Miron RJ, Bishara M, Choukroun J. Basics of platelet-rich fibrin therapy. Dentistry Today. 2017 Apr;36(4):74-76

[9] Clark D, Rajendran Y, Paydar S, et al. Advanced platelet-rich fibrin and freeze-dried bone allograft for ridge preservation: A randomized controlled clinical trial. Journal of Periodontology. 2018;89:379-387

[10] Shah R, Triveni MG, Thomas R, et al. An update on the protocols and biologic actions of platelet rich fibrin in dentistry. The European Journal of Prosthodontics and Restorative Dentistry. 2017;25:64-72

[11] Wend S, Kubesch A, Orlowska A, et al. Reduction of the relative centrifugal force influences cell number and growth factor release within injectable PRF-based matrices. Journal of Materials Science: Materials in Medicine. 2017;28:188 
[12] Tunalı M, Özdemir H, Küçükodacı Z, et al. A novel platelet concentrate: Titaniumprepared platelet-rich fibrin. BioMed Research International. 2014;2014:209548

[13] Wirohadidjojo YW, Budiyanto A, Soebono H. Platelet-rich fibrin lysate can ameliorate dysfunction of chronically UVA-irradiated human dermal fibroblasts. Yonsei Medical Journal. 2016;57(5):1282-1285

[14] Sclafani AP. Platelet-rich fibrin matrix for improvement of deep nasolabial folds. Journal of Cosmetic Dermatology. 2010;9:66-71

[15] Corso M, Vervelle A, Simonpieri A. Current knowledge and perspectives for the use of platelet-rich plasma (PRP) and platelet-rich fibrin (PRF) in oral and maxillofacial surgery. Part 1: Periodontal and dentoalveolar surgery. Current Pharmaceutical Biotechnology. 2012;13:1207-1230

[16] Khairy NM, Shendy EE, Askar NA, et al. Effect of platelet rich plasma on bone regeneration in maxillary sinus augmentation (randomized clinical trial). International Journal of Oral and Maxillofacial Surgery. 2013;42(2):249-255

[17] Döri F, Kovács V, Arweiler NB, et al. Effect of platelet-rich plasma on the healing of intrabony defects treated with an anorganic bovine bone mineral: A pilot study. Journal of Periodontology. 2009;80(10):1599-1605

[18] Simonpieri A, Corso M, Vervelle A. Current knowledge and perspectives for the use of platelet-rich plasma (PRP) and platelet-rich fibrin (PRF) in oral and maxillofacial surgery. Part 2: Bone graft, implant and reconstructive surgery. Current Pharmaceutical Biotechnology. 2012;13:1231-1256

[19] Wallace SS, Froum SJ. Effect of maxillary sinus augmentation on the survival of endosseous dental implants. A systematic review. Annals of Periodontology. 2003;8(1): 328-343

[20] Browaeys H, Bouvry P, De Bruyn H. A literature review on biomaterials in sinus augmentation procedures. Clinical Implant Dentistry and Related Research. 2007;9(3):166-177

[21] Choukroun J, Diss A, Simonpieri A, et al. Platelet-rich fibrin (PRF): A second-generation platelet concentrate. Part V: Histologic evaluations of PRF effects on bone allograft maturation in sinus lift. Oral Surgery, Oral Medicine, Oral Pathology, Oral Radiology, and Endodontics. 2006;101:299-303

[22] Pikos MA. Maxillary sinus membrane repair: Update on technique for large and complete perforations. Implant Dentistry. 2008;17:24-31

[23] Dohan Ehrenfest DM, Rasmusson L, Albrektsson T. Classification of platelet concentrates: From pure platelet-rich plasma (P-PRP) to leucocyte- and platelet-rich fibrin (L-PRF). Trends in Biotechnology. 2009;27(3):158-167 
[24] Mouhyi J, Dohan Ehrenfest DM, Albrektsson T. The peri-implantitis: Implant surfaces, microstructure, and physicochemical aspects. Clinical Implant Dentistry and Related Research. 2012 Apr;14(2):170-183

[25] Dohan Ehrenfest DM, Vazquez L. Pulling out, extraction or avulsion? Implant Dentistry. 2008;17(1):4

[26] Jang ES, Park JW, Kweon H. Restoration of peri-implant defects in immediate implant installations by Choukroun platelet-rich fibrin and silk fibroin powder combination graft. Oral Surgery, Oral Medicine, Oral Pathology, Oral Radiology, and Endodontics. 2010; 109(6):831-836 
